\section{As Regiões de Saúde no Brasil segundo internações: método para apoio na regionalização de saúde}

\author{
Health Regions in Brazil based on hospital \\ admissions: a method to support \\ health regionalization
}

\author{
Las Regiones de Salud en Brasil según \\ internamientos: método para el apoyo de la \\ regionalización en salud
}

Diego Ricardo Xavier 1

Ricardo Antunes Dantas de Oliveira 1

Christovam Barcellos 1

Raphael de Freitas Saldanha 1

Walter Massa Ramalho 2

Josué Laguardia 1

Francisco Viacava 1

doi: 10.1590/0102-311X00076118

\section{Resumo}

Este estudo aborda as regionalizações da saúde em várias escalas espaciais com base no fluxo de pacientes. Para isso, foram analisados dados por meio do relacionamento das informações de origem e destino das interações realizadas em nível municipal no Brasil em 2016. A análise tem como base a teoria dos grafos e utiliza um algoritmo de modularidade que busca agrupar municípios em comunidades que detêm grande número de conexões entre si. O algoritmo otimiza o número de entradas e saídas, levando em consideração o fluxo de pacientes. Os resultados são apresentados considerando diferentes estruturas espaciais politico-administrativas. Levando-se em conta o fluxo de pacientes sem restrições espaciais foram constituídas 29 comunidades no país, 64 comunidades quando respeitados os limites das grandes regiões e 164 considerando os deslocamentos apenas dentro dos estados. Os resultados demonstram a importância de regiões historicamente constituídas, desconsiderando limites administrativos, para a efetivação do acesso a serviços de saúde. Também revelam a aderência aos limites administrativos em muitas Unidades da Federação, demonstrando a importância dessa escala espacial no contexto do acesso às internações. A metodologia usada traz contribuições relevantes para o planejamento regional em saúde.

Regionalização; Sistemas de Informação Geográfica; Cidades 


\section{Introdução}

A regionalização do Sistema Único de Saúde (SUS) foi estabelecida como estratégia para otimizar a gestão do sistema, a racionalização de recursos e o aporte institucional para a criação de redes de atenção em saúde. Prevista na Constituição Federal de 1988, a regionalização registrou crescente importância no cenário nacional nos anos 2000, com a edição da Norma Operacional de Assistência à Saúde (NOAS 01/2002) 1 e a publicação do Pacto pela Saúde, em 2006 2. Em 2011, o Ministério da Saúde publicou o Decreto no 7.5083 que regulamenta a Lei no 8.080/1990 e, entre outras providências, orienta o processo de instituição das Regiões de Saúde.

Como destacam Duarte et al. 4 , as discussões sobre a regionalização no SUS são informadas tanto pela perspectiva de uma lógica que é endógena aos serviços de saúde e que tem como parâmetro o fluxo dos pacientes internamente ao sistema, quanto pelos debates acerca dos processos de distritalização que reconhecem a existência do território no entorno dos serviços de saúde e a constituição de áreas de abrangência e atuação destes serviços. Os determinantes da utilização dos serviços de saúde estão relacionados às necessidades de saúde, às características dos usuários e prestadores de serviços, e à organização do sistema de saúde. Dados os diversos níveis de complexidade, o acesso e uso dos serviços de saúde estão relacionados à oferta destes serviços e implicam padrões de fluxos de usuários nos territórios para o compartilhamento da rede de atenção à saúde.

Lima et al. 5 concluíram que a regionalização está associada às dinâmicas territoriais, às características do desenvolvimento econômico, às políticas de saúde, ao grau de articulação existente entre os representantes do poder público em saúde, e aos desenhos regionais adotados em cada estado. A complexidade da regionalização na saúde se deve a fatores como a institucionalização de uma rede homogênea de serviços e intervenções frente a uma grande heterogeneidade territorial, à formalização da responsabilidade pública e à regulação centralizada com a manutenção da autonomia dos governos locais 6 .

A análise de fluxos para a constituição de redes não é recente. Rabino \& Occelli 7 usaram informações de origem e destino de pacientes para classificar a rede considerando uma estrutura hierárquica. Outros trabalhos seguiram abordagens similares e buscaram entender como se estruturam as redes do ponto de vista da busca de atendimento 8,9,10. Apesar de caracterizar as redes de atendimento considerando procedimentos específicos ou caracterizando o processo de regionalização, poucos trabalhos têm apontado metodologias específicas capazes de propor a regionalização baseada nas redes observadas.

Este artigo apresenta como alternativa uma metodologia de configuração das Regiões de Saúde com base no deslocamento intermunicipal de pessoas para a utilização de serviços públicos ou conveniados ao SUS em diversas escalas espaciais político-administrativas. Para isso, é utilizada a teoria dos grafos e algoritmos de classificação que consideram modularidade, no intuito de identificar se a configuração atual das Regiões de Saúde conforma-se ao padrão de uso dos serviços hospitalares distribuídos no território nacional.

\section{Método}

Foram utilizados neste trabalho dados secundários obtidos de fontes de acesso público. A proposta deste estudo foi dispensada de apreciação por Comitê de Ética em Pesquisa, seguindo com os princípios da ética em pesquisa envolvendo seres humanos, constantes na Resolução no 510 do Conselho Nacional de Saúde (CNS), de 7 de abril de 2016.

Com base no local de residência e local de ocorrência das Autorizações de Internação Hospitalar (AIH), foi construída uma matriz de relacionamento de informações $n \times n$ na qual constam a origem do evento e o destino de realização da internação, utilizando-se o software Tabwin 4.0 (http://portal. saude.gov.br/portal/se/datasus/area.cfm?id_area=732). A análise foi realizada tendo como base o ano 2016, por ser o último período com dados consolidados e disponíveis no Sistema de Informações Hospitalares (SIH) até o momento. As informações foram obtidas no site do Departamento de Informática do SUS (DATASUS; http://www.datasus.gov.br). Com isso, obteve-se o número de interações entre municípios e o volume agregado total de deslocamentos para o período. Esses dados 
foram georreferenciados segundo as coordenadas das sedes municipais, com o objetivo de estimar as métricas de distância euclidiana média percorridas em cada grupo ou comunidade criada. A utilização da teoria de grafos para a análise de redes organizacionais permite a avaliação estrutural de um sistema de saúde 11. Dentre os recursos da análise de grafos, utiliza-se neste estudo a análise da modularidade aplicada a grafos, que possibilita expressar os deslocamentos em função dos locais de origem e de destino das internações hospitalares, considerando as limitações relacionadas às escalas espaciais. Em uma análise de redes é importante definir o que caracteriza os nós da rede e suas ligações, denominadas arestas. Neste estudo, os nós são os municípios de origem e destino dos pacientes que foram internados em determinado período e as arestas são determinadas pelo fluxo de pacientes entre estes municípios 12. Outra característica dessa rede é que as arestas são direcionais e ponderadas pelo volume de internações. Dessa forma, tem-se ao final um grafo direcional ponderado. Levando-se em conta que a rede de fluxos de pacientes entre os municípios varia segundo características locais, é esperada maior interação entre municípios da mesma localidade, constituindo comunidades ou módulos.

$\mathrm{Na}$ teoria de grafos, esses módulos são identificados, em geral, tomando-se a mutualidade das arestas, a sua ponderação e a menor distância entre os nós (proximidade matemática), o que permite a criação de diversos métodos com ênfase em diferentes princípios para a definição de módulos.

Neste artigo, adotou-se o método para a detecção de comunidades em grandes grafos que utilizam um algoritmo para grafos com pesos nas arestas 13 (o peso das arestas é entendido como o número de internações de indivíduos que se deslocaram em busca de atendimento). O método consiste na varredura por todos os vértices que são resultantes das combinações de nós e arestas. Dado um vértice $i$, calcula-se o ganho em modularidade com pesos 14 obtidos ao se colocar o vértice $i$ na comunidade de seu vizinho $j$, escolhendo aquela que, formada por um conjunto de municípios vizinhos, dá o maior aumento de modularidade (se aplicável). As novas comunidades são trocadas por supervértices, nos quais conexões entre estes vértices são arestas com peso igual à soma dos pesos nas arestas entre os vértices das mesmas. Esses passos são repetidos até que o valor que mede o grau de modularidade não possa mais ser melhorado 15 .

A função de modularidade aplicada a grafos resume vários aspectos das estruturas de comunidades em uma função baseada na comparação do grafo observado com um grafo aleatório, também chamado modelo nulo 14 . O conceito de modularidade mede a conexão de uma rede e a capacidade de se dividir em módulos ou comunidades. Nesse caso, maior modularidade exprime tendência da rede em agrupar municípios que detêm grande número de conexões entre si e pequeno número de conexões entre outros grupos de municípios. Desse modo, o algoritmo otimiza o número de entradas e saídas, levando em consideração o grau simples e o grau ponderado, ajustando um volume similar de entradas e saídas de internações. Considerando-se que o fluxo de pacientes é variável entre pares de municípios, a importância de cada aresta pode ser ponderada com base no quantitativo do fluxo.

O software Gephi 9.0.2 (https://gephi.org/users/download/) foi usado para o cálculo de modularidade, segundo três estratos geográficos que consideram os deslocamentos em todo o território nacional, em grandes regiões e dentro dos limites das Unidades da Federação. Desse modo, foram construídos 32 grafos: um grafo para todo o Brasil, cinco considerando as grandes regiões e 26 grafos para as Unidades da Federação. Nesses, o Distrito Federal, considerado um único vértice, foi incluído no Estado de Goiás para análise.

A expressão cartográfica da modularidade permite abordar as regiões constituídas pelo uso dos serviços de internação no país. Métodos para a deteç̧ão de módulos em redes são, em geral, não espaciais. Ou seja, não incluem ou pressupõem em suas lógicas a interação espacial e contiguidade das redes.

Com o objetivo de evidenciar as comunidades construídas com o algoritmo de modularidade e em observância às premissas que compõem uma Região de Saúde, como, por exemplo, a contiguidade espacial, as informações obtidas na análise de modularidade foram exportadas para o ambiente de Sistema de Informação Geográfica (SIG) e construídos mapas temáticos segundo as estratificações espaciais. Também foi possível extrair informações sobre a média e desvio padrão da distância euclidiana percorrida pelos pacientes. O software QGIS 3.0.2 (https://download.qgis.org/) foi usado para a elaboração dos mapas construídos segundo a base de municípios do ano de 2013, no formato Sistema de Referência Geocêntrico para as Américas (SIRGAS/2000) do Instituto Brasileiro de Geografia e 
Estatística (IBGE). Também foi usada a base geográfica de conformação das Regiões de Saúde para a comparação entre os resultados do modelo e a atual proposta de regionalização.

\section{Resultados}

No ano de 2016 foram registradas no Brasil 11.526.408 AIH, segundo dados coletados no SIH em dezembro de 2017. Mais de 67\% dessas internações ocorreram nos municípios de residência dos pacientes. O total de internações está distribuído em 92.478 interações municipais (arestas) e 96,5\% destas informações correspondem ao deslocamento intermunicipal de cerca de 3 milhões de internações.

Essas interações foram sistematizadas na análise de rede considerando-se os deslocamentos sem restrições dentro de todo o território nacional. O resultado do algoritmo de modularidade empregado resultou na criação de 29 comunidades, conforme apresentado na Figura 1a, na qual as cores do mapa dizem respeito ao conjunto de municípios (nós) e deslocamentos (aresta) que constituem uma comunidade ou módulo.

Na Figura 1b, observa-se a sistematização dos resultados em ambiente SIG, no qual se evidencia a classificação dos grupos de municípios segundo o fluxo de pacientes. Em parte das Unidades da Federação, as comunidades criadas seguem a conformação territorial do estado, com pouca variação em áreas limítrofes. Em outras áreas, as comunidades criadas não respeitam os limites territoriais dessas unidades.

Na Região Norte, por sua vez, observa-se três grandes áreas: uma incluindo os estados do Acre e Rondônia e sul do Estado de Amazonas, além de municípios a oeste do Mato Grosso; outra área inclui

\section{Figura 1}

Comunidades classificadas com o algoritmo de modularidade e distribuídas espacialmente, segundo internações no ano de 2016.

1a) Algoritmo de modularidade

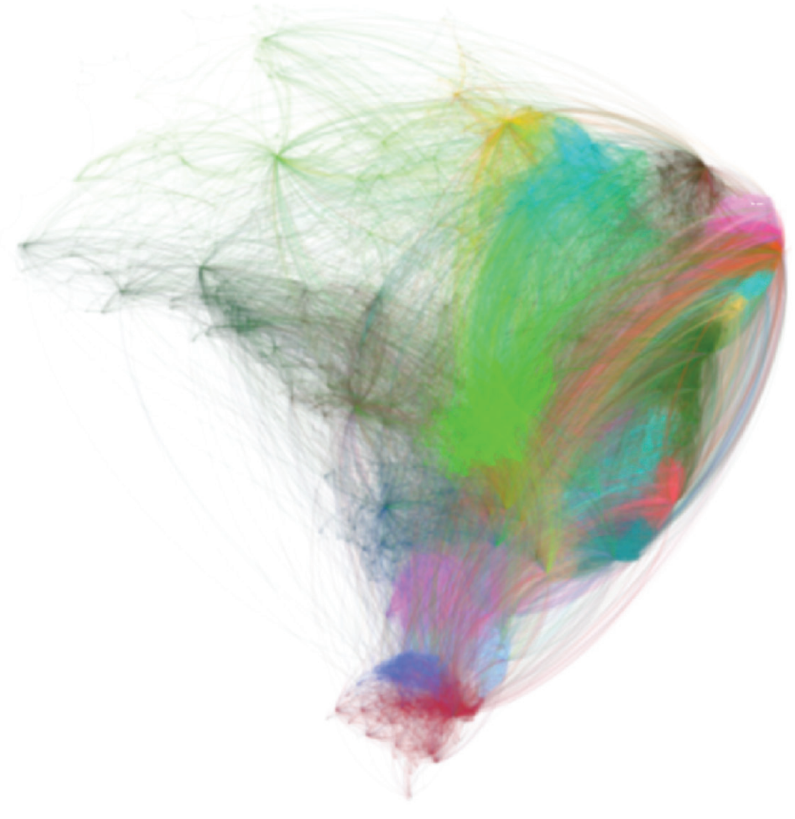

1b) Distribuição espacial

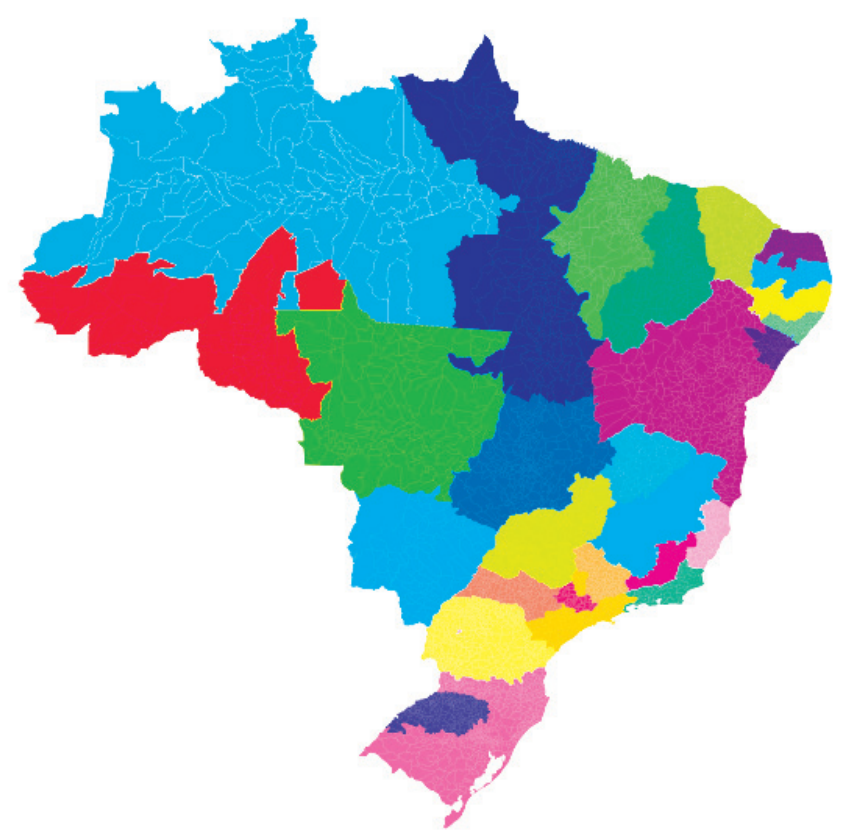


o Estado de Roraima, o restante do Amazonas e a parte oeste do Estado do Pará; a terceira área inclui o Estado de Tocantins, Amapá e leste do Pará.

No Nordeste, destaca-se o caso de Pernambuco, onde estão duas áreas distintas - uma a leste do estado e outra área a oeste, que apresenta trocas importantes com o Estado da Bahia, principalmente na região de Petrolina e Juazeiro. O mesmo ocorre entre municípios da Bahia e a região limítrofe do Estado de Alagoas. Parte do Estado do Maranhão é coberta pela comunidade do Piauí, principalmente nos arredores da cidade de Teresina.

Em Minas Gerais, observam-se cinco grupos distintos: norte do estado; Zona da Mata mineira; Vale do Jequitinhonha; Região Metropolitana de Belo Horizonte e Vale do Mucuri; Sul e Sudeste do estado. Além dessas áreas, municípios do noroeste do estado foram incluídos na comunidade que comporta o Estado de Goiás e o Distrito Federal.

Na Região Sudeste, o Estado de São Paulo apresentou quatro grandes regiões: a Região Metropolitana de São Paulo, que se estende até o Vale do Paraíba; o norte do estado, que forma uma única região com o Triângulo Mineiro; a Região Metropolitana de Campinas; e a região centro-oeste do estado.

No Sul do país, municípios limítrofes ao sul do Paraná foram incluídos no Estado de Santa Catarina. No Estado do Rio Grande do Sul, duas áreas distintas foram criadas, uma no noroeste do estado e a outra que abrange o restante dos municípios do estado.

A Tabela 1 apresenta os grupos criados e descritos nas Figuras 1a e 1b, segundo o número de municípios de cada comunidade, os graus de entrada e saída simples (interação ou fluxo, isto é, o número de conexões entre os municípios de cada comunidade) e ponderado (interação ou fluxo considerando como peso o número de internações), a população residente em cada grupo e o volume de internações em residentes.

\section{Tabela 1}

Graus de entrada e saída ponderados (aresta/internação) e simples (arestas/fluxos), municípios, interações em residentes e população das comunidades criadas, 2016.

\begin{tabular}{|c|c|c|c|c|c|c|c|c|c|c|c|c|}
\hline \multirow[t]{2}{*}{ Região } & \multicolumn{2}{|c|}{$\begin{array}{c}\text { Grau de entrada } \\
\text { ponderado } \\
\text { (internações) }\end{array}$} & \multicolumn{2}{|c|}{$\begin{array}{c}\text { Grau de saída } \\
\text { ponderado } \\
\text { (internações) }\end{array}$} & \multicolumn{2}{|c|}{$\begin{array}{c}\text { Grau de } \\
\text { entrada } \\
\text { (arestas/fluxos) }\end{array}$} & \multicolumn{2}{|c|}{$\begin{array}{c}\text { Grau de saída } \\
\text { (arestas/fluxos) }\end{array}$} & \multirow{2}{*}{$\begin{array}{l}\text { Municí- } \\
\text { pios } \\
\text { n }\end{array}$} & \multicolumn{2}{|c|}{$\begin{array}{l}\text { Internações em } \\
\text { residentes }\end{array}$} & \multirow{2}{*}{$\begin{array}{c}\text { População } \\
\text { n }\end{array}$} \\
\hline & $\mathbf{n}$ & $\%$ & $\mathbf{n}$ & $\%$ & $\mathbf{n}$ & $\%$ & $\mathbf{n}$ & $\%$ & & $\mathbf{n}$ & $\%$ & \\
\hline $\begin{array}{l}\text { Acre, Rondônia e } \\
\text { sul do Amazonas }\end{array}$ & 170.554 & 1,5 & 173.367 & 1,5 & 952 & 1,0 & 1.395 & 1,5 & 86 & 262.702 & 76,4 & 2.923 .133 \\
\hline $\begin{array}{l}\text { Amazonas/oeste } \\
\text { do Pará }\end{array}$ & 269.921 & 2,3 & 272.227 & 2,4 & 731 & 0,8 & 940 & 1,0 & 95 & 479.012 & 88,4 & 5.516 .113 \\
\hline $\begin{array}{l}\text { Amapá, leste do } \\
\text { Pará e Tocantins }\end{array}$ & 495.621 & 4,3 & 497.762 & 4,3 & 3.267 & 3,5 & 3.875 & 4,2 & 257 & 713.890 & 71,9 & 9.140 .677 \\
\hline Manaus & 385.643 & 3,3 & 385.874 & 3,3 & 4.706 & 5,1 & 4.104 & 4,4 & 215 & 540.646 & 70,1 & 6.422 .996 \\
\hline Piauí & 251.717 & 2,2 & 250.011 & 2,2 & 2.772 & 3,0 & 2.286 & 2,5 & 243 & 305.460 & 60,9 & 3.946 .825 \\
\hline Ceará & 476.305 & 4,1 & 476.048 & 4,1 & 2.232 & 2,4 & 2.307 & 2,5 & 184 & 625.424 & 65,7 & 8.963 .663 \\
\hline $\begin{array}{l}\text { Rio Grande do } \\
\text { Norte }\end{array}$ & 160.732 & 1,4 & 160.597 & 1,4 & 1.854 & 2,0 & 1.867 & 2,0 & 169 & 166.068 & 51,7 & 3.486 .569 \\
\hline Paraíba & 179.923 & 1,6 & 182.497 & 1,6 & 1.737 & 1,9 & 2.127 & 2,3 & 221 & 193.702 & 53,4 & 3.987 .844 \\
\hline $\begin{array}{l}\text { Leste de } \\
\text { Pernambuco }\end{array}$ & 489.171 & 4,2 & 479.292 & 4,2 & 4.154 & 4,5 & 3.356 & 3,6 & 160 & 390.230 & 40,3 & 8.418 .040 \\
\hline Alagoas & 175.197 & 1,5 & 176.370 & 1,5 & 1.042 & 1,1 & 1.273 & 1,4 & 99 & 192.788 & 54,8 & 3.324 .647 \\
\hline Sergipe & 104.588 & 0,9 & 105.299 & 0,9 & 1.004 & 1,1 & 1.074 & 1,2 & 90 & 83.680 & 39,9 & 2.577 .946 \\
\hline $\begin{array}{l}\text { Bahia, oeste de } \\
\text { Pernambuco }\end{array}$ & 854.623 & 7,4 & 860.670 & 7,5 & 7.904 & 8,5 & 8.011 & 8,7 & 430 & 1.214 .792 & 70,8 & 15.991 .011 \\
\hline $\begin{array}{l}\text { Norte de Minas } \\
\text { Gerais }\end{array}$ & 98.688 & 0,9 & 99.752 & 0,9 & 818 & 0,9 & 853 & 0,9 & 85 & 122.494 & 61,7 & 1.663 .125 \\
\hline
\end{tabular}

(continua) 
Tabela 1 (continuação)

\begin{tabular}{|c|c|c|c|c|c|c|c|c|c|c|c|c|}
\hline \multirow[t]{2}{*}{ Região } & \multicolumn{2}{|c|}{$\begin{array}{c}\text { Grau de entrada } \\
\text { ponderado } \\
\text { (internações) }\end{array}$} & \multicolumn{2}{|c|}{$\begin{array}{l}\text { Grau de saída } \\
\text { ponderado } \\
\text { (internações) }\end{array}$} & \multicolumn{2}{|c|}{$\begin{array}{c}\text { Grau de } \\
\text { entrada } \\
\text { (arestas/fluxos) }\end{array}$} & \multicolumn{2}{|c|}{$\begin{array}{l}\text { Grau de saída } \\
\text { (arestas/fluxos) }\end{array}$} & \multirow{2}{*}{$\begin{array}{c}\text { Municí- } \\
\text { pios } \\
\text { n }\end{array}$} & \multicolumn{2}{|c|}{$\begin{array}{l}\text { Internações em } \\
\text { residentes }\end{array}$} & \multirow{2}{*}{$\begin{array}{c}\text { População } \\
\text { n }\end{array}$} \\
\hline & $\mathbf{n}$ & $\%$ & $\mathbf{n}$ & $\%$ & $\mathbf{n}$ & $\%$ & $\mathbf{n}$ & $\%$ & & $\mathbf{n}$ & $\%$ & \\
\hline $\begin{array}{l}\text { Zona da Mata de } \\
\text { Minas Gerais }\end{array}$ & 158.871 & 1,4 & 160.027 & 1,4 & 1.681 & 1,8 & 1.745 & 1,9 & 152 & 197.248 & 61,9 & 2.391 .912 \\
\hline $\begin{array}{l}\text { Jequitinhonha, } \\
\text { Região } \\
\text { Metropolitana de } \\
\text { Belo Horizonte e } \\
\text { Vale do Mucuri } \\
\text { (Minas Gerais) }\end{array}$ & 628.345 & 5,5 & 624.256 & 5,4 & 4.946 & 5,3 & 5.154 & 5,6 & 377 & 789.108 & 63,0 & 11.368 .696 \\
\hline $\begin{array}{l}\text { Sul e sudoeste } \\
\text { de Minas Gerais }\end{array}$ & 172.282 & 1,5 & 175.907 & 1,5 & 2.170 & 2,3 & 2.420 & 2,6 & 149 & 223.176 & 64,1 & 2.774 .761 \\
\hline Espírito Santo & 235.887 & 2,0 & 236.275 & 2,0 & 1.918 & 2,1 & 1.892 & 2,0 & 80 & 241.984 & 51,3 & 4.003 .838 \\
\hline Rio de Janeiro & 675.609 & 5,9 & 676.009 & 5,9 & 2.902 & 3,1 & 2.641 & 2,9 & 92 & 1.053 .792 & 78,0 & 16.635 .996 \\
\hline $\begin{array}{l}\text { Centro-oeste de } \\
\text { São Paulo }\end{array}$ & 284.456 & 2,5 & 275.269 & 2,4 & 3.586 & 3,9 & 2.797 & 3,0 & 175 & 344.148 & 61,5 & 3.657 .805 \\
\hline $\begin{array}{l}\text { Norte de São } \\
\text { Paulo e Triângulo } \\
\text { Mineiro }\end{array}$ & 545.883 & 4,7 & 539.603 & 4,7 & 5.691 & 6,2 & 4.985 & 5,4 & 308 & 731.178 & 67,4 & 8.541 .074 \\
\hline $\begin{array}{l}\text { Sul } \\
\text { Metropolitana de } \\
\text { São Paulo e Vale } \\
\text { do Paraíba }\end{array}$ & 1.499 .910 & 13,0 & 1.501 .985 & 13,0 & 5.848 & 6,3 & 6.074 & 6,6 & 171 & 2.356 .482 & 78,5 & 29.102 .106 \\
\hline $\begin{array}{l}\text { Região } \\
\text { Metropolitana de } \\
\text { Campinas }\end{array}$ & 290.836 & 2,5 & 292.104 & 2,5 & 1.895 & 2,0 & 2.030 & 2,2 & 68 & 428.694 & 73,5 & 6.026 .588 \\
\hline Paraná & 832.212 & 7,2 & 830.523 & 7,2 & 8.375 & 9,1 & 8.080 & 8,7 & 411 & 979.648 & 58,9 & 11.504 .587 \\
\hline Santa Catarina & 450.031 & 3,9 & 452.192 & 3,9 & 5.980 & 6,5 & 6.379 & 6,9 & 284 & 519.128 & 57,5 & 6.650 .508 \\
\hline $\begin{array}{l}\text { Rio Grande do } \\
\text { Sul }\end{array}$ & 589.891 & 5,1 & 587.327 & 5,1 & 4.596 & 5,0 & 4.589 & 5,0 & 267 & 846.596 & 71,9 & 9.101 .429 \\
\hline $\begin{array}{l}\text { Noroeste do Rio } \\
\text { Grande do Sul }\end{array}$ & 165.184 & 1,4 & 168.427 & 1,5 & 2.355 & 2,5 & 2.892 & 3,1 & 228 & 200.648 & 60,1 & 2.178 .811 \\
\hline $\begin{array}{l}\text { Mato Grosso } \\
\text { do Sul }\end{array}$ & 164.514 & 1,4 & 167.055 & 1,4 & 937 & 1,0 & 1.250 & 1,4 & 79 & 272.404 & 82,2 & 2.682 .386 \\
\hline Mato Grosso & 190.703 & 1,7 & 191.716 & 1,7 & 1.945 & 2,1 & 2.202 & 2,4 & 136 & 269.784 & 70,5 & 3.265 .395 \\
\hline $\begin{array}{l}\text { Goiás, Distrito } \\
\text { Federal, noroeste } \\
\text { de Minas Gerais }\end{array}$ & 529.111 & 4,6 & 527.967 & 4,6 & 4.480 & 4,8 & 3.880 & 4,2 & 259 & 766.786 & 72,5 & 9.865 .586 \\
\hline Total & 11.526 .408 & 100,0 & 11.526 .408 & 100,0 & 92.478 & 100,0 & 92.478 & 100,0 & 5.570 & 15.511 .692 & 67,3 & 206.114 .067 \\
\hline
\end{tabular}

Em média, as comunidades apresentam 192 municípios. O grupo com o maior número (430) é o que abrange o Estado da Bahia e leste de Pernambuco, e a comunidade com o menor número de municípios é a Região Metropolitana de Campinas, no Estado de São Paulo, com 68 municípios.

Com relação ao número de internações, destaca-se o grupo de municípios no qual está incluída a Região Metropolitana de São Paulo, que comporta 13\% do total de internações. A região norte do Estado de Minas Gerais foi o grupo que apresentou o menor percentual de internações, 0,9\% do total, cerca de 100 mil internações no ano de 2016. Cabe ressaltar que em todas as comunidades a diferença percentual entre o grau de entrada e saída é similar, o que indica o nível de resolutividade das regiões criadas. 
A Figura 2 registra as regiões conformadas pelos deslocamentos dos pacientes contingenciados segundo as cinco grandes regiões do país, o que gerou 64 grupos. Na Região Norte, observam-se as áreas de influência dos estados de Rondônia e Acre no sul do Amazonas e do Estado de Roraima ao norte, assim como uma área de abrangência do Amapá e norte do Estado do Pará. Ainda no Pará, destacam-se a comunidade a leste do estado, a área Metropolitana de Belém, a área de Marabá e região sudeste do estado. O Tocantins apresenta duas comunidades distintas de influência nos deslocamentos.

No Nordeste, a maioria dos estados manteve o fluxo dentro dos seus limites territoriais, com pouca alteração em relação ao mapa anterior (Figura 1b). O Ceará apresenta três comunidades distintas, e na Bahia manteve-se a comunidade de Juazeiro e Petrolina, além de uma comunidade de municípios que abrange o centro sul do estado, uma que se estende de Salvador até o oeste do estado, e a região da costa do Cacau baiana.

\section{Figura 2}

Comunidades classificadas com o algoritmo de modularidade restrito às grandes regiões, segundo internações no ano de 2016.

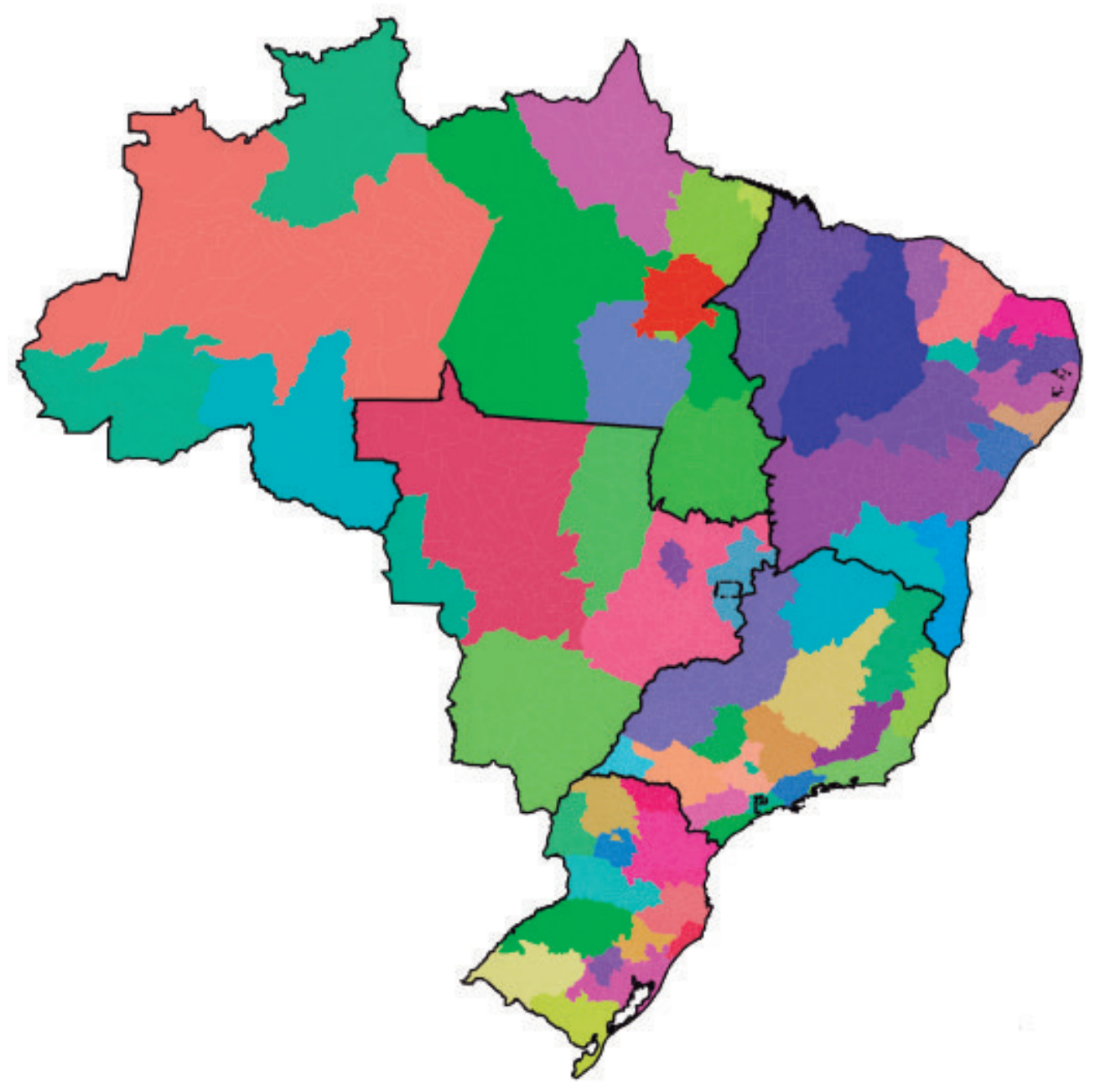


Na Região Centro-oeste, o Estado do Mato Grosso apresentou três comunidades, uma que vai do sul ao norte do estado, outra próxima à fronteira com a Bolívia e uma terceira a leste do estado. O Mato Grosso do Sul continua com uma única comunidade que abrange todo o estado. Em Goiás, destaca-se a comunidade com influência no entorno de Brasília e outra no centro norte do estado.

Na Região Sudeste, observam-se no Estado de São Paulo comunidades que englobam os municípios do entorno de Presidente Prudente, Ribeirão Preto, Bauru/Marília, São José do Rio Preto/ Barretos e o Triângulo Mineiro, além da região de São José dos Campos. Minas Gerais apresenta conformação similar à do mapa anterior (Figura 1), com exceção do comportamento na região noroeste do estado que passa a estar contida nos limites administrativos da Unidade da Federação. Os estados do Rio de Janeiro e Espírito Santo permanecem contidos nos limites da Unidade da Federação.

No Sul do país, no Estado do Paraná, foram registradas três regiões: a metropolitana de Curitiba; a porção oeste, que engloba Cascavel, Londrina e Maringá; e a região de Guarapuava. A região sudoeste do Paraná, no entorno da cidade de Pato Branco, foi incluída no grupo de municípios da região oeste de Santa Catarina. Ainda nesse estado, observa-se a área da região metropolitana de Florianópolis, Joinville ao norte e Criciúma ao sul. No Estado do Rio Grande do Sul, foram observadas cinco regiões: a metropolitana de Porto Alegre; Caxias do Sul ao norte; o entorno de Pelotas ao sul; um grupo de municípios no entorno de Passo Fundo, no noroeste do estado; além de um grupo na região de Santa Cruz do Sul e Santa Maria.

A Região Sudeste apresenta o maior volume de internações, seguida da Região Nordeste. Em média, as comunidades criadas nas regiões Sudeste e Nordeste apresentam cerca de 100 municípios em cada grupo; a Região Sul, 79; a Centro-oeste, 67; e na Região Norte, 38 municípios. É importante destacar que ao contrário das comunidades do Rio de Janeiro e Belo Horizonte que são compostas por muitos municípios, a comunidade criada no entorno do Município de São Paulo apresenta apenas 43 municípios.

A Figura 3 apresenta as comunidades criadas pelo algoritmo considerando-se os limites administrativos estaduais. A constituição das Regiões de Saúde segundo limites estaduais é adotada na configuração dos Planos Diretores de Regionalização (PDR) elaborados pelas secretarias estaduais de saúde. O algoritmo foi capaz de delimitar 164 Regiões de Saúde (Figura 3a), e a conformação atual conta com 438 Regiões de Saúde (Figura 3b).

A análise comparada entre as duas figuras possibilita evidenciar regiões similares tanto em sua constituição com base no PDR, quanto nos resultados estimados pelo modelo. Levando-se em conta que o modelo busca o equilíbrio entre o grau de entrada e de saída simples e ponderado e, por consequência, constitui comunidades que apresentam comportamento resolutivo, nessas regiões o deslocamento de pessoas que buscam atendimento no SUS parece respeitar o limite das regiões propostas no planejamento.

Na Região Norte, o sudoeste e a região do Município de Altamira, no Estado do Pará, o oeste do Estado do Acre, e o sul do Estado de Rondônia, em Vilhena, apresentaram aderência entre o proposto no PDR e o resultado da análise de grafos.

No Nordeste, o Estado do Ceará apresenta similaridade de área nos municípios de Itapipoca, Capistrano e Itapiúna; na Bahia, a Região Metropolitana de Salvador; no Piauí, o sul do estado e a região do Município de Parnaíba. Também apresentaram similaridades a área que abrange o município de Garanhuns em Pernambuco; as áreas dos municípios de São Bento, Brejo da Cruz e Guarabira, na Paraíba; e a área dos municípios de Ribeirópolis e Itabaiana em Sergipe, juntamente com a área sul do estado.

No Centro-oeste, destaca-se o Estado de Mato Grosso do Sul, que apresenta relativa correspondência entre as regiões propostas pelo PDR e as comunidades delimitadas pelo algoritmo. No entanto, o algoritmo foi capaz de discriminar três comunidades a mais na área próxima a Aquidauana. Em Mato Grosso, observa-se conformidade das áreas no entorno dos municípios de Rondonópolis, no sul do estado, e Apiacás, ao norte. Em Goiás, no entorno dos municípios de Santa Terezinha e Ceres, no centro do estado.

Na Região Sudeste, o sul do Estado do Espírito Santo; a região na área de Mogi-Guaçu em São Paulo; a região de Niterói e São Gonçalo; e a região serrana do Estado do Rio de Janeiro apresentam bastante similaridade. No Sul, foram aderentes as regiões do Município de Irati, no Paraná; Itajaí, Criciúma, Blumenau e Florianópolis, em Santa Catarina; e Erechim, no Rio Grande do Sul. 
Figura 3

Comunidades classificadas com o algoritmo de modularidade e Regiões de Saúde atuais, segundo internações no ano de 2016.

3a) Algoritmo de modularidade

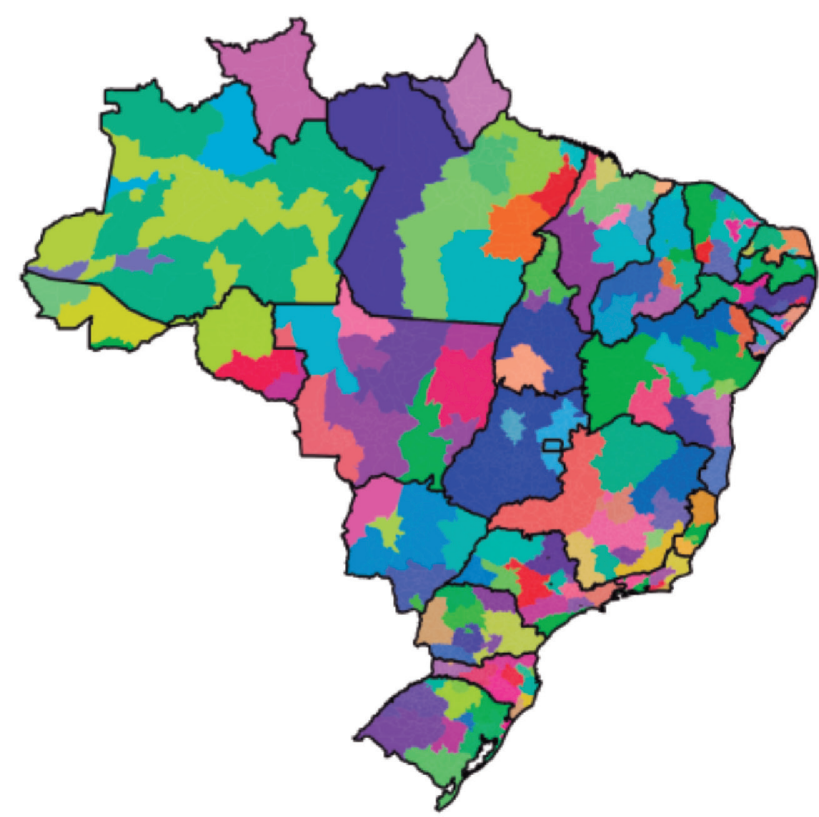

3b) Distribuição espacial

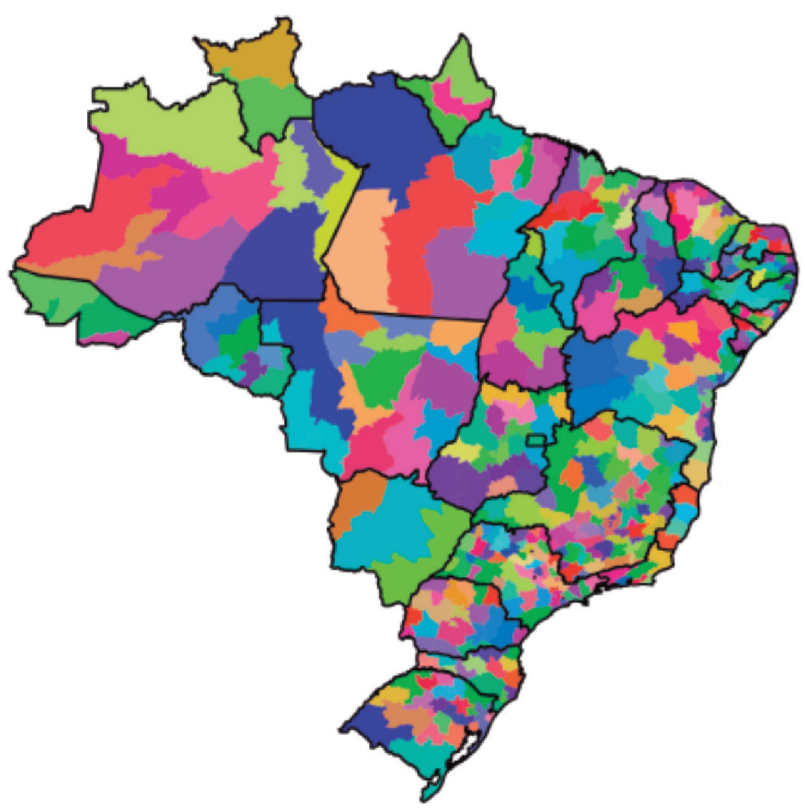

Além dessas áreas que apresentam delimitações semelhantes ou muito similares, em alguns casos o algoritmo unificou 2, 3 ou 4 regiões propostas pelo planejamento. É o caso de Minas Gerais, onde se observa conformação de áreas similares às Mesorregiões de Saúde do estado.

A Tabela 2 apresenta um resumo das comunidades criadas nos diferentes tipos de restrições geográficas adotadas, a distribuição das Regiões de Saúde em vigor segundo o PDR de cada UF e as médias e desvio padrão das distâncias de deslocamento para as comunidades, propostos pelo algoritmo.

Como descrito anteriormente, foram construídas 29 comunidades sem restrição político-administrativa. Tendo em vista a restrição de grandes regiões, foram criadas 64 comunidades e levando-se em conta a restrição aos estados foram geradas 164 comunidades.

Em todos os estados da Federação, o algoritmo previu um número menor de regiões quando comparado à configuração atual, com exceção de Mato Grosso do Sul, onde o algoritmo definiu 6 comunidades, acima do número total de três Regiões de Saúde existentes. As diferenças entre as Regiões de Saúde e as comunidades criadas pelo algoritmo são proporcionalmente maiores na medida em que aumenta a área político-administrativa, o que é esperado dado a proposta de otimização do algoritmo. Entretanto, considerando as restrições aos limites dos estados, Acre, Roraima, Amapá, Tocantins, Piauí, Sergipe, Espírito Santo e Rio de Janeiro apresentaram configurações dos grupos com as menores diferenças entre a configuração atual de Regiões de Saúde e as comunidades construídas com base na aplicação do algoritmo.

A média das distâncias percorridas foi maior nas 29 comunidades propostas sem a restrição político-administrativa, com média de $341 \mathrm{~km}$. Tendo em vista a restrição de grandes regiões, a menor distância percorrida observada ocorreu na Região Sul $(141 \mathrm{~km})$. Sudeste e Nordeste apresentaram distâncias médias similares, $159 \mathrm{~km}$ e $162 \mathrm{~km}$, respectivamente. Na Região Norte, com $235 \mathrm{~km}$, e na Região Centro-oeste, com 229km, observaram-se as maiores médias de distância. As distâncias também são discriminadas segundo Unidades da Federação, e apesar de ser razoável a avaliação de que a 


\section{Tabela 2}

Número de municípios, média e desvio padrão da distância percorrida, Regiões de Saúde e comunidades criadas com o algoritmo de modularidade, considerando as restrições territoriais do país, grandes regiões e Unidades da Federação.

\begin{tabular}{|c|c|c|c|c|}
\hline & Municípios & Regiões de Saúde & Comunidades & $\begin{array}{c}\text { Distância média } \\
\text { (desvio padrão) em km }\end{array}$ \\
\hline Brasil & 5.570 & 438 & 29 & $341,34(531,24)$ \\
\hline Grandes regiões & - & - & 64 & - \\
\hline Unidades da Federação & - & - & 164 & - \\
\hline Norte & 450 & 45 & 12 & $235,32(285,44)$ \\
\hline Rondônia & 52 & 7 & 3 & $155,39(132,40)$ \\
\hline Acre & 22 & 3 & 3 & $173,55(195,70)$ \\
\hline Amazonas & 62 & 9 & 4 & $300,43(349,20)$ \\
\hline Roraima & 15 & 2 & 1 & $100,89(87,56)$ \\
\hline Pará & 144 & 13 & 8 & $153,95(169,67)$ \\
\hline Amapá & 16 & 3 & 2 & $127,03(100,88)$ \\
\hline Tocantins & 139 & 8 & 5 & $171,24(143,22)$ \\
\hline Nordeste & 1.794 & 133 & 14 & $162,82(196,14)$ \\
\hline Maranhão & 217 & 19 & 8 & $136,79(120,60)$ \\
\hline Piauí & 224 & 11 & 8 & $139,97(135,61)$ \\
\hline Ceará & 184 & 22 & 8 & $109,23(115,17)$ \\
\hline Rio Grande do Norte & 167 & 8 & 4 & $92,96(86,70)$ \\
\hline Paraíba & 223 & 16 & 5 & $94,47(100,09)$ \\
\hline Pernambuco & 185 & 12 & 5 & $111,43(116,55)$ \\
\hline Alagoas & 102 & 10 & 5 & $67,07(51,17)$ \\
\hline Sergipe & 75 & 7 & 4 & $58,02(34,61)$ \\
\hline Bahia & 417 & 28 & 10 & $139,77(139,88)$ \\
\hline Sudeste & 1.668 & 153 & 16 & $159,21(167,57)$ \\
\hline Minas Gerais & 853 & 77 & 12 & $131,71(132,16)$ \\
\hline Espírito Santo & 78 & 4 & 4 & $89,7(64,43)$ \\
\hline Rio de Janeiro & 92 & 9 & 6 & $78,42(60,27)$ \\
\hline São Paulo & 645 & 63 & 12 & $131,21(119,90)$ \\
\hline Sul & 1.191 & 68 & 15 & $141,17(134,26)$ \\
\hline Paraná & 399 & 22 & 8 & $157,16(135,22)$ \\
\hline Santa Catarina & 295 & 16 & 11 & $111,49(110,07)$ \\
\hline Rio Grande do Sul & 497 & 30 & 11 & $109,44(105,67)$ \\
\hline Centro-oeste & 467 & 39 & 7 & $229,43(238,39)$ \\
\hline Mato Grosso do Sul & 79 & 4 & 6 & $153,78(135,82)$ \\
\hline Mato Grosso & 141 & 16 & 8 & $263,64(213,59)$ \\
\hline Goiás & 246 & 18 & 3 & $138,69(115,07)$ \\
\hline Distrito Federal * & 1 & 1 & - & - \\
\hline
\end{tabular}

* Incluído no Estado de Goiás.

distância esteja associada à área da unidade de análise, na Região Norte observa-se comportamento inverso no Acre; no Nordeste, Bahia e Piauí apresentam distâncias similares, assim como Minas Gerais e São Paulo, no Sudeste. 


\section{Discussão}

Este estudo propôs um método capaz de auxiliar na discussão sobre o processo de regionalização considerando os deslocamentos das populações em busca de atendimento. Essa questão vai além de entender como os fluxos e as redes de atendimento se configuram. Alguns trabalhos buscam discutir e debater esse problema $8,9,12$, pois é necessário entender quais são as interações entre os municípios que embasam esse deslocamento.

Esse comportamento tem origens que não se limitam apenas à oferta e demanda de serviços, mas também estão relacionadas à constituição do espaço geográfico, suas redes de transporte e as relações entre as populações desses municípios 16, à articulação entre regiões historicamente estabelecidas e às regionalizações, que são ferramentas instrumentais à atuação de agentes do Estado e do setor privado 17. Por exemplo, a coincidência, em diversos casos, dos limites das comunidades geradas pelo algoritmo e o território dos estados pode revelar o papel ainda preponderante deste ente federativo na regulação das internações. Em outros casos, as comunidades formadas se assemelham a regiões tradicionalmente reconhecidas pela população, como o Pampa Gaúcho, o Triângulo Mineiro, a Baixada Santista ou regiões de recente povoamento, como o sul do Amazonas e o norte dos estados do Acre e Rondônia.

A Resolução no 1, de 29 de setembro de 2011 18, que estabelece diretrizes gerais para a instituição de Regiões de Saúde, nos termos do Decreto no 7.508, de 28 de junho de 2011 3, define que: "A Região de Saúde configura o espaço geográfico contínuo constituído por agrupamento de municípios limítrofes, delimitado a partir de identidades culturais, econômicas e sociais e de redes de comunicação e infraestrutura de transportes compartilhados, com a finalidade de integrar a organização, o planejamento e a execução de ações e serviços de saúde". Essa proposta pode ser observada com base no uso dos serviços de saúde, entretanto, os planos diretores por vezes têm de se adequar a três questões práticas: arranjos políticos, administrativos e orçamentários. Nesse sentido, é possível que a conformação atual das Regiões de Saúde se preste a atender exclusivamente a esses três componentes, deixando em segundo plano a rotina das pessoas e a história dos lugares, o que acaba por limitar o que propõe a legislação. As contradições verificadas na prática também estão relacionadas ao fortalecimento dos colegiados regionais, com preposições de medidas para evitar a fragmentação dos sistemas regionais, ao mesmo tempo em que no nível local busca solidificar o governo e a sua permanência 19.

As Figuras 1 e 2 exemplificam as regiões constituídas ao longo do tempo, e embora existam os limites administrativos, que constituem e caracterizam lugares como as Unidades da Federação, em algumas áreas, sobretudo nas fronteiras estaduais, ocorreu conformação de comunidades onde se confundem estes limites. Do ponto de vista da geografia humana, levando-se em conta o espaço do cotidiano das populações, as fronteiras administrativas também não são espaços de contenção, o que consequentemente reflete nas interações e deslocamentos e na utilização dos serviços de saúde.

A região de Petrolina e Juazeiro é exemplo de um importante polo interestadual de produção e comércio de frutas 20,21 . Se por um lado a região detém status de polo conjunto na produção e circulação de mercadorias e seus limites político-administrativos não interferem na vida das pessoas, por outro, a organização das Regiões de Saúde foi baseada nos limites estaduais, seguindo critérios que podem atender a questões de cunho político, administrativo e orçamentário, mas que desconsideram a constituição histórica, o cotidiano e a conformação do espaço geográfico. Contudo, devem-se considerar as complicações envolvidas em pactuações interfederativas, e frente a este fato propor mecanismos que auxiliem nestas questões.

Outras áreas descritas na Tabela 1 e na Figura 2 consideram as delimitações espaciais das grandes regiões do Brasil e destacam áreas como o Triângulo Mineiro e o norte de São Paulo, Acre/Rondônia e o sul do Amazonas, Tocantins e Pará, entre outras, que são regiões conhecidas e tradicionais na conformação histórica do país 22 revelam assim, lógicas de uso dos serviços de saúde que não se restringem a limites estaduais, facilitadas por vias de transporte que as conectam.

A análise da rede composta pelos deslocamentos permitiu uma nova abordagem sobre possíveis reconfigurações da regionalização atual por meio de recortes baseados no uso dos serviços de saúde, desde uma delimitação macrorregional até estadual, que não estão limitados apenas às definições de Região de Saúde dos estados. Os resultados mostraram que foi definida uma menor quantidade de Regiões de Saúde do que as atualmente definidas, apontando para a necessidade de se considerar 
áreas com abrangência mesorregional na saúde, especialmente quando se leva em consideração procedimentos com maior grau de complexidade. Outro ponto importante é que o número de Regiões de Saúde atualmente proposto provavelmente comporta regiões que apresentam baixo grau de resolutividade para a assistência hospitalar, que pode ser devido ao escasso número de leitos disponíveis em unidades de pequeno porte ou à ausência de hospitais. O simples cumprimento da norma imposta na conformação regional de saúde, sem levar em consideração tanto os padrões de uso quanto o rol de ações e serviços de saúde disponíveis à população das regiões, desvirtua o que é proposto no Decreto no 7.508 .

Uma vantagem da metodologia adotada neste estudo é que as redes foram construídas exclusivamente com base no deslocamento populacional, sem interferência político-administrativa ou qualquer juízo de caráter subjetivo. A avaliação dos resultados desta proposta pode apontar áreas onde a regionalização está possibilitando o acesso de forma adequada à população dos municípios que compõem a região, bem como espaços que constituiriam vazios assistenciais. Os resultados encontrados requerem análises complementares, que levem em conta grupos de procedimentos com níveis variados de complexidade, assim como a distribuição dos recursos físicos e humanos dos serviços de saúde do país e os dados do sistema privado de saúde. Os achados desses novos estudos podem subsidiar a discussão do processo de regionalização da saúde, especialmente no que tange ao papel dos determinantes socioeconômicos e da estruturação dos serviços de saúde. Nesse sentido, as grandes desigualdades regionais existentes no país não se restringem ao acesso a serviços de saúde, mas é também resultado de desigualdades de renda, educação e condições de transporte, sua disponibilidade e custo, que vão por sua vez propiciar maior ou menor mobilidade da população em busca destes serviços.

A relação entre o ajustado pelo algoritmo na entrada e saída de pacientes é muito semelhante. Nesse sentido, a área descrita pelo algoritmo e posteriormente espacializada no mapa sugere regiões nas quais não é necessário ou é muito pequeno o volume de deslocamento de pacientes para outros grupos de municípios. Essa característica demonstra um elevado grau de autonomia dos grupos formados, o que sugere uma relativa integração interna de redes e a presença de polos no seu interior que atraem serviços e demandas de uma determinada área de influência 22 . Essa é uma importante premissa de resolutividade da Região de Saúde, considerando que muitas dessas regiões têm grande extensão territorial, como é o caso da Região Norte.

Ressaltamos limitações inerentes relacionadas ao uso de dados secundários do SIH, portanto, os dados podem estar subestimados. É importante também ressaltar que este estudo representa dados transversais do ano de 2016, por isto, passíveis de alterações nos demais anos. Contudo, a validade dos resultados não é comprometida, tendo em vista que os fluxos são influenciados por processos sociohistóricos, não somente pela situação atual. Também o uso da distância linear entre sedes de cidades nem sempre corresponde às condições de deslocamento locais, uma vez que as redes de transporte terrestres não alcançam grande parte do país, principalmente nas regiões Norte e Centro-oeste. Outra questão importante de ser destacada é que não foi avaliada a complexidade dos procedimentos realizados, deste modo, as regiões apresentadas se restringem ao volume de internações, e de acordo com o nível de complexidade outras conformações devem ser observadas, fato que não invalida os achados deste artigo, mas sugere desdobramentos desta análise.

O presente trabalho buscou se apoiar nos dados de uso dos serviços que, de certa maneira, sintetizam questões adjacentes, como acesso geográfico e disponibilização de equipamentos e de recursos humanos. Propõe-se aqui um instrumento metodológico que ajuda a orientar o processo de regionalização, tendo em vista as redes de atendimento existentes e o uso dos serviços, tanto para conformação quanto para a avaliação das Regiões de Saúde propostas. Nesse sentido, esse instrumento deve ser considerado, em conjunto com outras questões de ordem orçamentária, política e administrativa, sem que se sobreponham, mas que dialoguem com aspectos históricos, contextuais e cotidianos das populações para as quais as Regiões de Saúde devem atender. 


\section{Colaboradores}

D. R. Xavier concebeu o estudo, coletou os dados, construiu mapas, analisou os resultados e elaborou o texto. R. A. D. Oliveira concebeu o estudo, analisou os resultados e elaborou o texto. C. Barcellos, R. F. Saldanha, W. M. Ramalho, J. Laguardia e F. Viacava analisaram os resultados e contribuíram na elaboração do texto.

\section{Informações adicionais}

ORCID: Diego Ricardo Xavier (0000-0001-52597732); Ricardo Antunes Dantas de Oliveira (00000003-0144-7288); Christovam Barcellos (00000002-1161-2753); Raphael de Freitas Saldanha (0000-0003-0652-8466); Walter Massa Ramalho (0000-0001-5085-5670); Josué Laguardia (00000003-1456-5590); Francisco Viacava (0000-00031486-2157).

\section{Agradecimentos}

Agradecemos a toda equipe do projeto PROADESS (Projeto de Avaliação de Desempenho do Sistema de Saúde).

\section{Referências}

1. Ministério da Saúde. Portaria no 373, de 27 de fevereiro de 2002. Diário Oficial da União 2002; 27 fev.

2. Ministério da Saúde. Portaria no 399, de 22 de fevereiro de 2006. Divulga o Pacto pela Saúde 2006: consolidação do SUS e aprova as Diretrizes Operacionais do Referido Pacto. Diário Oficial da União 2006; 23 fev.

3. Ministério da Saúde. Decreto no 7.508, de 28 de junho de 2011. Regulamenta a Lei no 8.080, de 19 de setembro de 1990, para dispor sobre a organização do Sistema Único de Saúde SUS, o planejamento da saúde, a assistência à saúde e a articulação interfederativa, e dá outras providências. Diário Oficial da União 2011; 29 jun.

4. Duarte LS, Pessoto UC, Guimarães RB, Heimann LS, Carvalheiro JR, Cortizo CT, et al. Regionalização da saúde no Brasil: uma perspectiva de análise. Saúde Soc 2015; 24:472-85.

5. Lima LD, Vianna ALd'A, Machado CV, Albuquerque MV, Oliveira RG, Iozzi FL, et al. Regionalização e acesso à saúde nos estados brasileiros: condicionantes históricos e político-institucionais. Ciênc Saúde Colet 2012; 17:2881-92.

6. Vianna ALd'A, Lima LD, Ferreira MP. Condicionantes estruturais da regionalização na saúde: tipologia dos Colegiados de Gestão Regional. Ciênc Saúde Colet 2010; 15:2317-26.

7. Rabino GA, Occelli S. Understanding spatial structure from network data: theoretical considerations and applications. Cybergeo 1997; (29):4-9.

8. Oliveira EXG, Melo ECP, Pinheiro RS, Noronha $\mathrm{CP}$, Carvalho MS. Acesso à assistência oncológica: mapeamento dos fluxos origemdestino das internações e dos atendimentos ambulatoriais. O caso do câncer de mama. Cad Saúde Pública 2011; 27:317-26.

9. Grabois MF, Oliveira EXG, Carvalho MS. Assistência ao câncer entre crianças e adolescentes: mapeamento dos fluxos origem-destino no Brasil. Rev Saúde Pública 2013; 47:368-78.

10. Albuquerque MV, Vianna ALd'A. Perspectivas de região e redes na política de saúde brasileira. Saúde Debate 2015; 39(n. especial):28-38.

11. Luke DA, Harris JK. Network analysis in public health: history, methods, and applications. Ann Rev Public Health 2007; 28:69-93.

12. Sousa LMO, Araújo EM, Miranda JGV. Caracterização do acesso à assistência ao parto normal na Bahia, Brasil, a partir da teoria dos grafos. Cad Saúde Pública 2017; 33:e00101616.

13. Blondel VD, Guillaume JL, Lambiotte R, Lefebvre E. Fast unfolding of communities in large networks. J Stat Mech 2008; 2008:P10008.

14. Newman MEJ. Analysis of weighted networks. Phys Rev 2004; 70(5 Pt 2):056131.

15. Machado FM. Detecção de comunidades em grafos multicamada muito grandes [Dissertação de Mestrado]. Belo Horizonte: Departamento de Ciência da Computação, Universidade Federal de Minas Gerais; 2016. 
16. Santos M. A natureza do espaço: técnica e tempo - razão e emoção. 3a Ed. São Paulo: Editora Hucitec; 1996.

17. Ribeiro ACT. Regionalização: fato e ferramenta. In: Limonad E, Haesbaert R, Moreira R, organizadores. Brasil, século XXI: por uma nova regionalização? Agentes, processos e escalas. São Paulo: Max Limonad; 2004. p. 194-212.

18. Ministério da Saúde. Resolução no 1, de 29 de setembro de 2011. Estabelece diretrizes gerais para a instituição de Regiões de Saúde no âmbito do Sistema Único. Diário Oficial da União 2011; 29 set.

19. Mello GA, Pereira APCM, Uchimura LYT, Iozzi FL, Demarzo MMP, Viana ALA. O processo de regionalização do SUS: revisão sistemática. Ciênc Saúde Colet 2017; 22:1291-310.
20. Camelo Filho JV. A dinâmica política, econômica e social do Rio São Francisco e do seu vale. Revista do Departamento de Geografia 2011; 17:83-93

21. Silva PCG. Articulação do interesse público e privado no Pólo Petrolina-PE/Juazeiro-BA. Em busca de espaço no mercado globalizado de frutas frescas [Tese de Doutorado]. Campinas: Universidade Estadual de Campinas; 2001.

22. Corrêa RL. Interações espaciais. In: Castro IE, Gomes PC, Corrêa RL, organizadores. Explorações geográficas. Rio de Janeiro: Bertrand Brasil; 1997. p. 279-318. 


\section{Abstract}

This study addressed health regionalization on various spatial scales based on patient flow. The article analyzed data through data linkage on the origin and destination of admissions at the municipal level in Brazil in 2016. The analysis is based on graph theory and uses a modularity algorithm that seeks to group municipalities in communities with a large number of interlinks. The algorithm optimizes the number of hospital admissions and discharges, taking patient flow into account. The results are shown, considering different political and administrative spatial structures. Considering patient flow without spatial restrictions, 29 communities were created in the country, compared to 64 communities when the boundaries of the major geographic regions were respected, and 164 when considering only patient flows within the respective states. The results show the importance of historically constituted regions, ignoring formal administrative boundaries, in order to implement access to health services. They also reveal adherence to administrative boundaries in many states of Brazil, demonstrating this spatial scale's importance in the context of access to hospital admissions. The methodology makes relevant contributions to regional health planning.

Regional Health Planning; Geographic Information Systems; Cities

\section{Resumen}

Este estudio aborda las regionalizaciones en salud dentro de varias escalas espaciales, basadas en el flujo de pacientes. Para tal fin, se analizaron datos a través de la relación existente entre la información de origen y destino, procedente de interacciones realizadas a nivel municipal en Brasil durante 2016. El análisis está basado en la teoría de los grafos y utiliza un algoritmo de modularidad que busca agrupar municipios en comunidades que cuentan con un gran número de conexiones entre sí. El algoritmo optimiza el número de entradas y salidas, teniendo en consideración el flujo de pacientes. Los resultados se presentan considerando las diferentes estructuras espaciales politico-administrativas. Considerando el flujo de pacientes sin restricciones espaciales, se constituyeron 29 comunidades en el país, 64 comunidades respetando los limites de las grandes regiones, $y$ 164 considerando desplazamientos sólo dentro de los estados. Los resultados demuestran la importancia de las regiones históricamente constituidas, desconsiderando limites administrativos, para hacer efectivo el acceso a servicios de salud. También revelan la adherencia a los limites administrativos en muchas Unidades Federales, demostrando la importancia de esta escala espacial en el contexto del acceso a los internamientos. La metodología utilizada aporta contribuciones relevantes para la planificación regional en salud.

Regionalización; Sistemas de Información

Geográfica; Ciudades
Recebido em 16/Abr/2018

Versão final reapresentada em 10/Dez/2018

Aprovado em 14/Dez/2018 